\title{
TEM Study of Structure and Deformation Mechanism of Superplastic Titanium Based Alloy
}

\author{
A. Kumao and K. Nishio
}

Kyoto Institute of Technology, Dept. of Electronics and Information Science

Matsugasaki, Sakyo-ku, Kyoto 606-8585, Japan

Titanium alloys are very useful materials because they have excellent properties such as high strength and tough corrosion resistance, especially in cold forming and heat-treatment. The sample studied in this paper is a Ti-22V-4Al alloy, which has the properties of superplasticity. In pure Ti metal $\alpha$-phase(hcp) is stable at a temperature below $882^{\circ} \mathrm{C}$, while $\beta$-phase(bcc) is stable at a temperature above $882^{\circ} \mathrm{C}$. Vanadium atoms added to pure Ti metal stabilize $\beta$-phase, while aluminum atoms stabilize $\alpha$-phase.[1] In this paper, the superplastic deformation mechanism of this sample was examined by means of $200 \mathrm{kV}$ TEM and EDS in relation to the structure.

Fig.1(a) shows the electron diffractogram of the original sample and Fig.1(b) is the dark field image taken with the beams indicated by arrows in (a). Moiré pattern mainly consisted of $\alpha$-Ti $(10 \cdot 0)$ and $\beta$-Ti(10-1) is recognized, where the distance between two adjacent fringes is $2.7 \mathrm{~nm}$, corresponding to the theoretical distance. High-resolution bright field image around the moire fringe is shown in Fig.1(c), in which hexagonal patterns are seen as indicated by arrows. They show the structure image of $\alpha$-Ti crystal projected onto the $(00 \cdot 1)$ plane. Figs.2(a) and (b) show Ti atom positions projected along [00.1] for $\alpha$-phase and [111] for $\beta$-phase, respectively. The orientation relation between $\alpha$-phase and $\beta$-phase is found to be $\alpha-\operatorname{Ti}(00 \cdot 1)[10 \cdot 0] / / \beta-\operatorname{Ti}(111)[2-1-1]$.

Fig. 3 show the electron micrograph of the sample elongated by a stress of $60 \mathrm{Mpa}$ during heating at $800^{\circ} \mathrm{C}$, where the strain was $250 \%$. The granular contrast is caused by $\alpha$-Ti phase, which has a tendency to precipitate along the slip lines arose perpendicular to the direction of a stress. On the other hand, smooth area is constituted of $\beta$-Ti phase. These conclusions were derived by comparing the two EDS spectra obtained from the granular area and from the smooth area in Fig.3. An example of EDS spectrum measured at the smooth area is shown in Fig.4. The intensity of the vanadium atoms obtained from the smooth area is relatively higher than that obtained from the granular area. This means that at the smooth area there are more vanadium atoms added to stabilize $\beta$-phase than at the granular area. Moreover, big disturbance of lattice fringes was not recognized in the deformed image. The reason is in the fact that there is no distortion inside the deformed sample because slips occur at the special planes on both $\alpha$ - and $\beta$-phases.[2] Fig.5 shows the schematic diagram showing how to slip. In this alloy, two phases coexist by keeping the special orientation relation mentioned above. In this case, the special slip planes and slip directions are $(00 \cdot 1)$ and [10 -0] for $\alpha$-Ti crystal, and (110) and [11-1] for $\beta$-Ti crystal. Thus, since this material is subject to slip along almost the same direction, large amount of deformation can be obtained without defects and/or lattice distortion.

References

[1] H.W. Rosenberg, Journal of Metals, 30 (1983) 455.

[2] R.C. Gifkins, Met. Trans. A, Vol.7A (1976) 1225. 

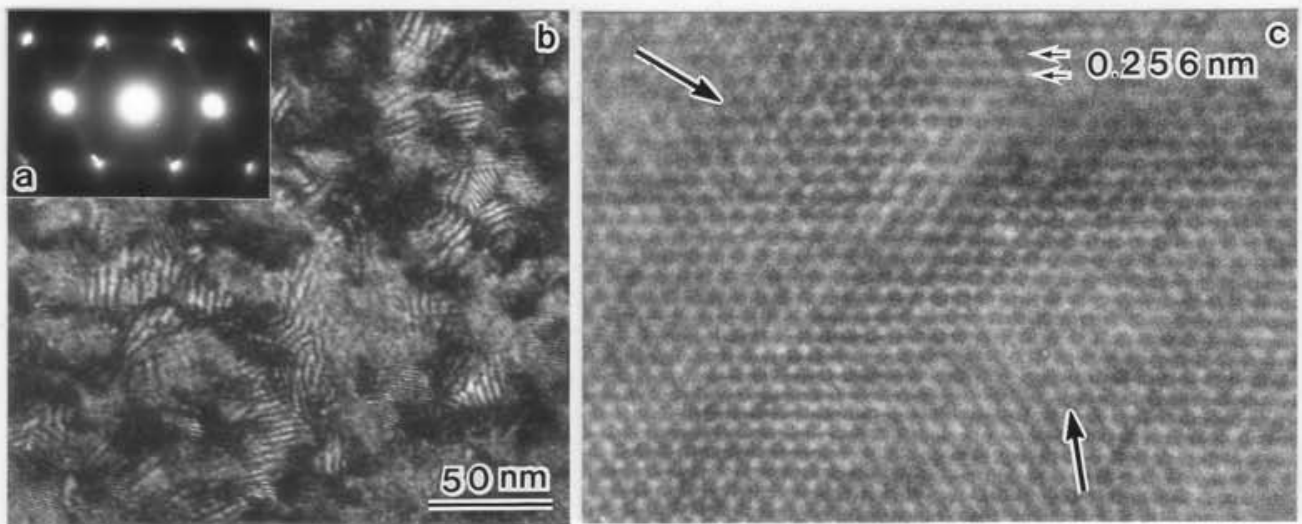

FIG. 1. Electron diffractogram (a) and dark field image(b) of a Ti-V-Al alloy, in which $\alpha$-and $\beta$-phases of Ti crystals coexist. They form moiré fringes. (c) Structure image of $\alpha$-Ti crystal observed along the c-axis.

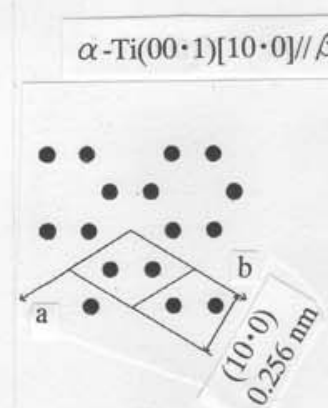

a

FIG. 2. Ti atom positions projected onto $(00 \cdot 1)$ for $\alpha$-Ti crystal (a) and (111) for $\beta$-Ti (b).

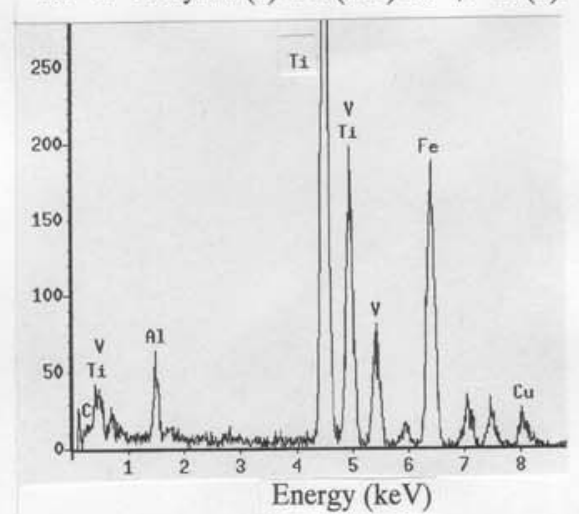

FIG. 4. EDS spectrum obtained from the smooth area in Fig.3.

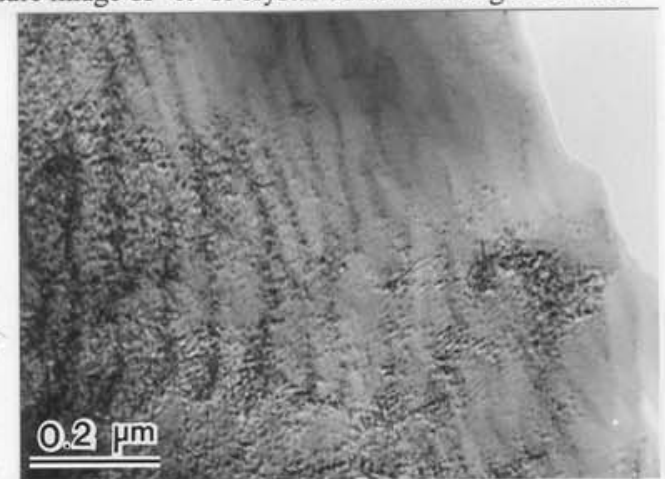

FIG. 3. Image of a deformed sample heated at $800 \mathrm{C}$. Wavy contrast shows slip lines normal to the stress.

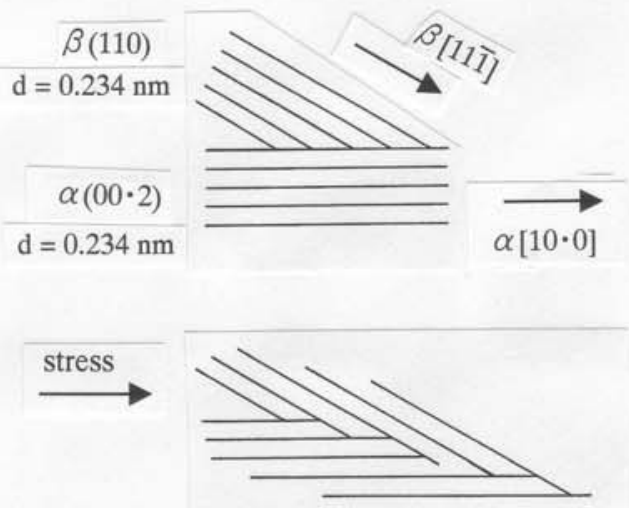

FIG. 5. Schematic drawing of the slip model. 